lingt, bei denen aber auch die Gefahr der Allgemein- und Herdreaktionen am größten ist.

Ich resümiere somit: Das Vitaltuberkulin ist ein Präparat, das nicht regelmäßig lebensfähige menschliche Tuberkelbacillen enthält, aber alle Bestandteile des Tuberkelbacillus, in schonendster Form aufgeschlossen, in sich birgt. Es steht in seinem therapeutischen Effekt der Kochschen Bacillenemulsion nahe. Ich sehe nach den Erfahrungen mit diesem Präparat die Frage, ob eine Behandlung mit lebenden, schwach virulenten humanen Bacillen der Tuberkulinbehandlung überlegen ist, noch nicht als im negativen Sinne entschieden an.

\section{WIE LANGE UND IN WELCHER KONZENTRATION VERBLEIBT SALVARSAN IM MENSCHLICHEN BLUT? \\ Von}

Hans Th. Schreus und Alfred Holländer.

Aus der Universitäts-Hautklinik Bonn, (Direktor: Prof. ERICH HOFFMANN.)

In Verfolg von Utberlegungen, die über die Wirkungsweise des Salvarsans und eine etwaige Verbesserung des therapeutischen Erfolges angestellt wurden und über die an anderer Stelle berichtet werden soll, erschien es uns von grundlegender Bedeutung, das Konzentrationsgefälle des Salvarsans im menschlichen Blutserum festzustellen.

Verschiedene Autoren haben sich mit dieser wichtigen Frage besonders in den ersten Jahren nach Einführung des Salvarsans beschäftigt.

F. BLUMEN'Thal ${ }^{1}$ ) konnte am Kaninchen nachweisen, da $\beta$ das Arsen nach Einspritzung von Atoxyl noch nach I6 Stunden im Blute in erheblicher Menge zu finden ist.

IGERSHEIMER und ROTHMANN ${ }^{2}$ ) fanden mit Hilfe der von BLUMENTHAL ausgearbeiteten Diazoreaktion, da $\beta$ das Atoxyl nach intravenöser Injektion im Kaninchenblut 5 -I 5 Minuten, sogar $2^{1 / 2}$ Std. nach der Injektion in unverändertem $Z$ ustand nachweisbar ist.

Nach FISCHER und HoPPE ${ }^{3}$ ) scheint das Salvarsanpräparat bei intravenöser Injektion aus dem Blut zur gleichen Zeit wie aus Urin und Darm zu verschwinden. Am 2. Tage konnte Arsen nachgewiesen werden. Nach I4 Tagen negatives Resultat.

BorNSTEIN ${ }^{4}$ ) wies nach, da $\beta$ bei einem Kaninchen $I_{1} 1 / 2$ Stunde post inj. schon der größte Teil des Präparats aus dem Blute verschwunden war.

STUMPKE und SIEG.FRIED ${ }^{5}$ ) konnten höchstens am I. oder 2. Tage mäßige Mengen von As im Blute nachweisen.

LöHLEIN ${ }^{6}$ ) stellte fest, daß bei Kaninchen nach intravenöser Injektion bis 24 Stunden post inj. der As-Nachweis im Blut positiv ausfiel.

HEUSER ${ }^{7}$ ) fand nach einer am 28. I. I922 erfolgten intravenösen Injektion am 8. III. I922 im Blut noch Arsen.

FISCHER und ZERNICK ${ }^{8}$ ) konnten nach intravenöser Injektion mehrere Monate lang As im Blut nachweisen.

Nach BuRNASCHOFF ${ }^{9}$ ) zirkulieren im Blut in den ersten 24 Stunden etwa $9 \%$ des eingeführten Arsens.

ABELIN ${ }^{10}$, der den Salvarsannachweis im Blut bei 8 Patienten geführt hat, fand eine positive Reaktion bis I Stunde post inj., nach $I^{1 / 2}$ Stunde ein $z$ weifelhaftes und nach $2^{1 / 2}$ Stunden ein negatives Ergebnis.

RTEBES $^{11}$ ) wies im Kaninchenblut I $_{5}$ Minuten bis 40 Stunden post inj. Arsen nach. - Beim Menschen fiel die Probe 3 Stunden post inj. negativ aus. Bei den Patienten, bei denen in 2 Tagen 3 Injektionen gemacht wurden und nach der 3. Injektion untersucht wurde, war die Resorcinreaktion im Serum noch nach 4 Stunden positiv.

Aus obiger Zusammenstellung ergibt sich zunächst die Tatsache, daß von den verschiedenen Untersuchern außerordentlich voneinander abweichende Resultate gefunden wurden. Eine solche Verschiedenheit erklärt sich sofort aus den mannigfachen Untersuchungsmethoden. Während die einen das Salvarsanmolekül als solches zur Grundlage ihrer Prüfung benutzten, beschränkten sich andere darauf, nur das Arsen nachzuweisen. Es ist verständlich, daB bei dem raschen Zerfall des unstabilen Salvarsanmoleküls im Körper große Differenzen zutage treten mußten. Tatsächlich findet man auch, daß die Untersucher, die sich auf den Nachweis des Arsen verlegten, länger positive Ausschläge hatten als diejenigen, die das Salvarsan als solches nachwiesen. Da die chemotherapeutische Wirksamkeit des Salvarsans nach EHRLICHs Anschauungen zweifellos vom unversehrten Salvarsanmolekül abhängig ist, sind für unsere Zwecke die mit dem reinen As-Nachweis erzielten Daten von minderer Bedeutung. Es bleiben also lediglich die Untersuchungen nach der Ehrlich-Bertheim schen ${ }^{12}$ ) (Pardimethylamidobenzaldehyd) und der Abelinschen ${ }^{13}$ ) Methode. Die von uns in der riesigen Salvarsanliteratur zerstreut hier und dort gefundenen Ergebnisse, von denen die wichtigsten oben zitiert sind, sind aber zu lückenhaft und geben nicht genügend Anhaltspunkte, um den genauen Verlauf des Konzentrationsabfalls im Blutserum nach einer oder mehreren Injektionen $\mathrm{zu}$ bestimmen. Außerdem sind diese Untersuchungen fast ausschließlich an Tieren vorgenommen worden, und es erscheint uns nicht erlaubt, daraus ohne weiteres auf menschliche Verhältnisse zu schließen.

Wir haben deshalb bei einer Anzahl von Kranken erneut Untersuchungen angestellt und systematisch in kurzen Zeiträumen nach intravenösen Injektionen der verschiedenen Salvarsanpräparate und Dosen Blutproben entnommen.

Nach orientierenden Vorarbeiten entschlossen wir uns die von ABELIN ${ }^{13}$ ) angegebene Methode, an dessen Vorschriften wir uns im allgemeinen hielten, unseren Prüfungen zugrundezulegen. Die Probe beruht auf der Diazotierung des Salvarsans durch verdünnte Salzsäure und $1 / 2$ proz. Natriumnitritlösung und späterem Hinzufügen einer durch $\mathrm{Na}_{2} \mathrm{CO}_{3}$ alkalisch gemachten Resorcinlösung. Bei Anwesenheit von Salvarsan entsteht ein schöner roter Farbstoff, der deutlich von etwa bestehender Eigenfärbung der zu untersuchenden Flüssigkeit zu unterscheiden ist. - Wir haben niemals unspezifische Resultate bei Kontrollversuchen gehabt. Die Methode ist außerordentlich empfindlich und gibt bei geeigneter Versuchsanordnung bis $z u$ einer Verdünnung von I : I00 000 deutlich erkennbare Ausschläge.

Es empfiehIt sich, um die bei hohen Verdünnungen schwächeren Färbungen deutlich sichtbar zu machen, die zu untersuchende diazotierte Flüssigkeit über die alkalische Resorcinlösung zu schichten. Grundbedingung für einwandfreie Resultate sind absolut reine und farblose Reagenzien; Soda- und Resorcinlösung soll möglichst jedesmal frisch bereitet werden, da schon nach kurzem Stehen sich Verfärbungen einstellen. Außerđem sind alle zu benutzenden Gefäße und Pipetten peinlichst sauber zu halten. Zweckmäßig wird die Uberschichtung in möglichst engen Röhrchen $(z$. B. im unteren engen Teil von Zentrifugenröhrchen) vorgenommen, was auch den Vorteil hat, daß man mit geringen Mengen auskommt.

Bei sorgfältiger Technik gelingt es, die Farbstoffabstufungen der Konzentrationen von I: 1000 bis I: 100 000 so deutlich $\mathrm{zu}$ gestalten, daß eine einwandfreie Stufenskala gewonnen wird, Letzteres ist von besonderer Bedeutung, da es sich hier um quantitative Feststellungen handelt. [Vgl. B. Barker, BeEson und P. G. ALBRECHT ${ }^{14}$ )]. Besonders schöne Skalen erhielten wir be Verwendung von Neosilber-Salvarsan-Natrium.

Wir haben, wie auch ABEIIN, beobachtet, daB öfter nach kurzem Warten von I-2 Minuten die Färbung deutlicher hervortritt. Die Farben halten sich aber besonders in den höheren Verdünnungen nur kurze Zeit einwandfrei, so daß bei jeder Untersuchung von Blutserum eine neue Skala hergestellt werden mußte. Das Ablesen der Farbtönungen gelingt am besten gegen einen hell erleuchteten weißen Hintergrund (FlieBpapier!) und zwar nur bei Tageslicht. Lampenbeleuchtung ist unbrauchbar. - $3-5 \mathrm{ccm}$ Blut, das zentrifugiert wird, geben reichlich Serum zur Vornahme der Untersuchung. Geringe Eigenfärbungen der Seren stören nicht.

Wenn den so gewonnenen quantitativen Werten auch keine absolute Genauigkeit zukommt, so dürfte die Fehlergrenze $20-30 \%$ nicht überschreiten. Die relativen Zahlen der einzelnen Versuche aber, die also die Natur des Kurvenverlaufs bestimmen, haben zweifellos eine noch größere Genauigkeit.

Im folgenden mögen zunächst die Resultate folgen, die wie durch Blutserumuntersuchungen auf Salvarsangehalt bei $3^{\mathrm{r}}$ Luetikern gewonnen haben. Die Untersuchungen erstreckten sich auf die Feststellung der Anwesenheit von Salvarsan im Blutserum überhaupt, auf die Abschätzung der Höhe der jeweils vorhandenen Konzentration, auf das Verhalten der Höhe der Einzelgaben zum Konzentrationsabfall und schließlich auf den Einfluß etwaiger vorhergegangener Salvarsaninjektionen auf den Konzentrationsabfall.

I. Was die Feststellung der Anwesenheit im Blutserum überhaupt anlangt, so konnten wir bald die Angaben früherer 
Beobachter (ABeliN u. a.) bestätigt finden. Länger als 3 Stunden post inj. konnten wir in keinem Falle noch Salvarsan im Blutserum nachweisen, wenigstens nicht in Konzentrationen, die oberhalb der Empfindlichkeitsschwelle unserer Untersuchungsmethode lagen. Wenn wir trotzdem bei einigen Patienten auch noch bis zu 6 Stunden nach der Injektion untersuchten, so geschah es lediglich aus dem Grunde, um festzustellen, ob nicht auch eine wechselnde Konzentration im Blute vorkommt, wie sie etwa durch schubweises Abgeben des Salvarsans aus den Geweben und etwaigen Speicherungsstätten (Leber, Milz, Herz, Lunge) in die Blutbahn zustande kommen könnte. Diese Ansicht findet man ab und $z u$ in der Literatur vertreten. Ein solches Vorkommen findet in Wirklichkeit aber nicht statt, wie die folgenden Versuchsprotokolle zeigen.

I. Pat. Bo. Lues I.

24. V. I922, IO ${ }^{25}$ a. m.: I Inj. von Salv.-Natr. 0,45 . Nach 30 Min. Salvarsankonzentration im Blutserum: $\therefore \quad 2$ Std. roMin: Salvarsankonzentration i. Blutserum: +

2. Pat. Ni. Lues II.

24. V. 1922. $10^{25}$ a. m.: I Inj. von Salv.-Natr, 0,45 . Nach 25 Min.: +

3. Pat. Kü. Lues I. I. Inj. von Neo-Salv. o,45 am I 5. V. 1922. ig. V. 1922 $10^{25}$ a. m. 2. Inj. von Neo-Salv. 0,6

Nach I5 Min.: +

r Std. 5 Min.: +

"2 Std. 5 Min.: +.

4. Pat. Schi. Lues II. 2 Inj. von Neo-Salv. 0,45 u. 0,6 vom 26. V. bis 29. V. 1922.

2. VI. I 922 Io Uhr a. m.: 3. Inj. von Neo-Salv. 0,6. Nach I Std.: +.

" 3 , : +.

5. Pat. Ha. Lues II. 2 Inj. von Neo-Salv, 0,3 u, 0,45 . vom 27. V. bis 30 . V. 1922

2. VI. I922 $9^{55}$ a. m.: 3. Inj. von Neo-Salv. 0,45 .

Nach I Std.: + .

" 3 , : +.

6. Pat. We. Lues II. 2 Inj. von Neosilber-Salv.-Natr. 0,2 u. 0,3 vom 24. V. bis 29. V. I922.

2. VI. I $9229^{50}$ a. m.: 3. Inj. von Neosilber-Salv.-Natr, 0,4 . Nach I Std.: + . , 3 , : + .

7. Pat. Bo. Lues I. 2 Inj. von Salv.-Natr. 0,45 vom 24 . V. bis 29. V. 1922. (Subchronische Glomerulonephritis.)

9. VI. I922. 3. Inj. von Salv.-Natr. 0,45 . Nach $3 \frac{1}{2}$ Std.: -

8. Pat. Gr. Lues II. 3 Inj. von Neo-Salv. $0,3-0,45-0,6$ vom 6. V. bis $15 . \mathrm{V}$. I922.

I9. V. I922 $\mathrm{TO}^{25}$ a. m. : 4. Inj. von Neo-Salv. 0,6.

Nach I 5 Min.: + .

"I Std. 5 Min. : t.

" 2 Std. 5 Min.: +.

9. Pat. We. Lues II. (Siehe Nr. 6.)

6. VI. 1922 4. Inj. von Neosilber-Salv.-Natr. 0,45 . Nach 6 Std.: -

ro. Pat. Schi. Lues II. (Siehe Nr. 4.) 6. VI. 1922 4. Inj. von Salv.-Natr. 0,6. Nach 6 Std.: -..

II. Pat. Ha. Lues IT. (Siehe Nr. 5.) 6. VI. I922 4. Inj. von Neo-Salv. 0,45 . Nach 6 Std.: -

I2. Pat. Nie. Lues II. 3 Inj. von SaIv.-Natr. $0,3-0,45-0,6$ vom 24. V. bis 2. VI. 1922.

6. VI. I922 4. Inj. von Salv.-Natr. 0,45 .

Nach 6 Std.: -

13. Pat. Bo. Lues I. (Siehe Nr. 7.)

13. VI. $19229^{40}$ a. m.: 4. Inj. von Salv.-Natr. 0,6.

Nach $21 / 2$ Std.: 一.

, 3 Std.: -

"3 Std. Io Min. : -

I4. Pat. Mö. Lues II. 3 Inj. von Neosilber-Salv.-Natr. 0,2-0,3 bis 0,4 vom 21 . VI. bis 26 . VI. 1922 . 30. VI. I 922 4. Inj. von Neosilber-Salv.-Natr. 0,45 Nach 4 Std.: -

" 5 , : -
15. Pat. Schri. Lues II. 4. Inj. von Neosilber-SaIv.-Natr. 0,45 vom I6. VI. bis 26. VI. 1922.

30. VI. I 922 5. Inj, von Neosilber-Salv.-Natr. 0,45 .

Nach 4 Std.

$$
\text { " } 5 \text {, : - - }
$$

16. Pat. Mi. Lues II. 7 Inj. von Neosilber-Salv.-Natr. 0,3-0,4 $-0,4-0,5-0,4-0,4$ u. Salv.-Natr. 0,6 vom 21. IV. bis I9. V. I922.

24. V. 1022 I0 $^{20}$ a. m.: 8. Inj. von Salv.-Natr. 0,6.

Nach 30 Min.: + .

"2 Std. Io Min.: + .

17. Pat. Nie, Lues II. 8 Inj. von Salv.-Natr. $0,3-0,45-0,6$ $-0,45-0,45-0,45-0,45-0,45$ vom 24 . V. bis 23 . VI. 1922. 26. VI. I922 9. Inj. von Salv.-Natr. 0,45.

Nach I Std. 45 Min.: Salv,-Konzentration im Blutserum: 1: 80000 .

18. Pat. Ha. Lues II. 8 Inj. von Neo-Salv, $0,3-0,45-0,45$ $-0,45-0,45-0,45$ u. Salv.-Natr. $0,45-0,45$ vom $27 . \mathrm{V}$ bis 23 . VI. IO22.

26. VI. $19229^{50}$ a. m.: 9. Inj. von Salv.-Natr. 0,45.

Nach I Std. 45 Min.: I : 70000.

19. Pat. Ha. (Siehe Nr. I8.)

3o. VI. I922 $9^{45}$ a. m.: Io. Inj, von Salv.-Natr. 0,45 . Nach 3 Std.: I : 80-90 000.

20. Pat. Nie. (Siehe Nr. I7.) 30. VI. I $9229^{47}$ : Io. Inj. von Salv.-Natr. $0,45$.

Nach 3 Std.: $x$ : 80-90 ooo.

II. War mithin in keinem Falle länger als 3 Stunden nach Injektion die Anwesenheit von Salvarsan im Blutserum nachzuweisen, so galt es zu prüfen, in welcher Form sich der Konzentrationsabfall im einzelnen vollzog. Zu diesem $Z$ weck entnahmen wir in kurzen Zeiträumen nach der Injektion (von I5 zu I5 Minuten) Blutproben, deren Salvarsankonzentration nach oben beschriebener Technik bestimmt wurde. Auch hierbei trugen wir Sorge, daB sowohl bezüglich der Höhe der Einzelgaben wie auch bezüglich der Anzahl der vorhergegangenen Injektionen möglichste Vielfältigkeit herschte.

Um den Verlauf des Konzentrationsabfalls möglichst anschaulich zu machen, haben wir die gefundenen Werte zum Teil in graphischer Darstellung beigefügt. Die Kurven wurden so gewonnen, daß auf der Ordinatenachse die Konzentrationen, als Abszissen die Zeiten nach der Injektion eingetragen wurden.

21. Pat. Lis. Lues II.

3o. VI, $19229^{50}$ a. m.: 1. Inj. von Salv.-Natr. 0,3.

Nach I5 Min.: $1: 40000$.

" 30 ": $x: 50000$.

"60 " : $1: 70000$.

22. Pat. Mö. Lues II. I. Inj. von Neosilber-Salv.-Natr. 0,2 am 2I. VI. Ig22.

23. VI. $19229^{48}$ a. m.: 2. Inj. von Neosilber-Salv,-Natr. 0,3 .

Nach is Min.: I : 30000 .

" 30 ": $1: 40000$

" $45 \quad$ ": $1: 50000$.

"60 " : $1: 70000$.

23. Pat. Schr. Lues II. I Inj. von Salv.-Natr. 0,45 am I6. VI. r.922.

19. VI. 1922 IO ${ }^{12}$ a. m.: 2. Inj. von Salv.-Natr. 0,45 .

Nach I9 Min.: I : 40000 .

, 34 ": I : 60000

" $48, ":$ : $1: 70000$

" 63 ", : I : 70000.

24. Pat. Mö. (Siehe Nr. 22.)

26. VI. $19229^{55}$ a. m.: 3. Inj. von Neosilber-Sa.-Na.:0,4.

Nach I $48^{\prime}:$

$\left.\begin{array}{lll}\prime & 2 & 5^{\prime}: \\ \| & 2 & 37^{\prime}:\end{array}\right\}$ I : 80000

25. Pat. Scbr. (Siehe Nr. 23.)

3. Inj. von Neosilber-Salv.-Natr. 0,45 am 23 . VI. I922. 26. VI. $19229^{57}$ a. m.: 4. Inj. von Neosilber-Salv.-Natr. 0,45 .

Nach I $43^{\prime}:$ I : $70-80000$.

" $25^{\prime}: 1: 70-80000$.

"2 $38^{\prime \prime}:$ I: $: 80000$.

26. Pat. We. (Siehe Nr. 6 u. 9.)

I4. VI. $192210^{27}$ a. m.: 5. Inj, von Neosilber-Salv.Natr. 0.45

Nach I 5 Min.: I : 30 000.

" 30 " : $x: 60000$.

$" 45,: 1: 70000$

" $60,: 1: 70-80000$. 
27. Pat. Ni. (Siehe Nr. I7.)

19. VI: 1922 I0 ${ }^{22}$ a, m.: 7. Inj. von Salv.-Natr. 0,45 ,

Nach I6 Min.: I : $30-40000$.

" $\quad 3 \mathrm{I} \quad, \quad:$ I $: 50000$.

". 56 , : I : 60-70000.

28. Pat. Ha. (Siehe Nr. I8.)

19. VI. $192210^{06}$ a. m.: 7. Inj. von Salv-Natr.. 0,45.

Nach I 5 Min.: 1 : 30000 .

" 30, : $1: 50000$.

" $45, \quad:$ I $: 60000$.

"60 " : I : 70000 .

29. Pat. Sch. (Siehe Nr. Io.)

Vom Io. VI. bis I4. VI. 1922 weitere 2 Inj. von Neo-Salv. $0,6-0,6$.

I9. VI. 1922 I0 17 a. m. : 7. Inj. von Salv.-Natr. 0,6.

Nach 30 Min.: 1 : 50000 .

$, \quad 45, \quad: 1: 70000$.

"60 ,.: : $\mathrm{x}: 80000$.

30. Pat. El. Lues II. 2 Inj. von Neosilber-Salv.-Natr. o, 3 u. 0,4 am 7. VII. u. Io. VII. 1922.

14. VII. Ig22 10 ${ }^{05}$ a. m.: 3. Inj. von Neo-Salv. o,6.

Nach I 5 Min.: $x: 30000$.

" 30 , : $1: 50000$.

, $45, ":$ I : 60000

, 60, : $1: 70000$.

31. Pat. Mö. (Siehe Nr. 14.)

Weitere 3 Inj. von Neosilber-Salv.-Natr. 0,45 vom 3. VI. bis Io. VII. 1922

14. VIr. Ig22 $10^{02}$ a. m.: 8. Inj. von Neo-Salv. 0,6 .

Nach I5 Min.: I : 30000.

" $30 \quad$, : I $: 50000$.

, 45, : $:$ I : 60000.

, 60, : I : 70000.

Aus diesen Kurven ist das wohl am meisten beachtenswert, daß der Konzentrationsabfall sich durchaus ähnelt. Die Kurven sind von einer Regelmäßigkeit, daß ein beinahe ge-
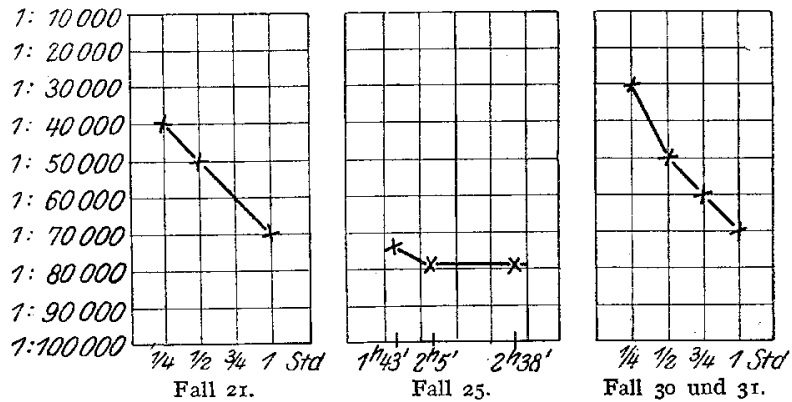

setzmäßiges Abfallen der Salvarsankonzentration im Blute als wahrscheinlich zu verzeichnen ist. Hier scheinen also individuelle Anlagen von sehr geringer Bedeutung zu sein, sonst würden wir bei der nicht kleinen Anzahl untersuchter $\mathrm{Pa}$ tienten die eine oder andere Abweichung gefunden haben. Bei der Literaturdurchsicht haben wir zudem auch nur einen Fall [RIEBES $\left.{ }^{11}\right)$ finden können, bei dem noch nach I9 Stunden Salvarsan im Blute nach der Abelin schen Methode nachzuweisen war. Kommen mithin gröbere individuelle Schwankungen nicht vor, so liegen natürlich kleinere von Mensch zu Mensch durchaus im Bereich der Möglichkeit. Auch unsere Kurven lassen ja solche kleinen Unregelmäßigkeiten erkennen, die, wenn sie nicht durch Versuchsungenauigkeiten bedingt sein sollten, in der Natur des einzelnen begründet sein müssen. Praktisch dürften solche Ausnahmen wohl nur dann Bedeutung erlangen, wenn es sich durch weitere Untersuchungen zeigen sollte, daß Menschen mit verzögertem Konzentrationsabfall zu Intoxikationserscheinungen (Dermatitis, Encephalitis) neigen. (Endothelschädigung?)

III. Was die Beziehung der Höhe der Einzelinjektionen zum Konzentrationsabfall betrifft, so haben wir hier keine merklichen Unterschiede bei Dosen $0,3-0,6 \mathrm{~g}$ feststellen können. Die Anzahl der untersuchten Personen ist aus äußerlichen Gründen leider nicht groß genug, um eine sichere Verallgemeinerung dieses Resultats zu gestatten.

IV. Bemerkenswert ist ferner, daß ein besonders augenfälliger Unterschied im Konzentrationsabfall bei der Prüfung nach der I. Injektion wie auch nach mehreren voraufgegangenen Injektionen nicht $\mathrm{zu}$ konstatieren ist. Das bedeutet nichts anderes, als daß die Faktoren, die für die Entnahme des Salvarsans aus dem Blute in Betracht kommen, und auf deren Natur wir hier nicht eingehen wollen, durch die vorhergehenden Injektionen in keiner merklichen Weise verändert worden sind. Diese Tatsache ist um so beachtenswerter, als die klinische Erfahrung den Gedanken einer kumulierenden Wirkung mehrerer in kurzen Abständen sich folgenden Injektionen nahelegt. Erblickt man eine solche in der allmählichen Sättigung der Gewebe mit Salvarsan, so müßte wohl nach mehreren Injektionen der Konzentrationsabfall langsamer erfolgen. Hierfür ergeben sich keine Stützpunkte.

V. Die von uns untersuchten Salvarsanpräparate (Neosalvarsan, Salvarsannatrium, Neosilbersalvarsannatrium) lassen keine merkliche Verschiedenheit in der Schnelligkeit des Konzentrationsabfalls erkennen. Inwieweit diese Tatsache mit der ziemlich gleich starken therapeutischen Wirkung dieser Präparate in Zusammenhang steht, sei dahingestellt.

VI. Letzten Endes dürfte noch die Frage interessieren, in welcher Weise die Schnelligkeit des Konzentrationsabfalls zahlenmäßig zum Ausdruck kommt. Zur Klärung dieser Frage sind die Kurven der Fälle 2I-3I aufeinander gezeichnet,

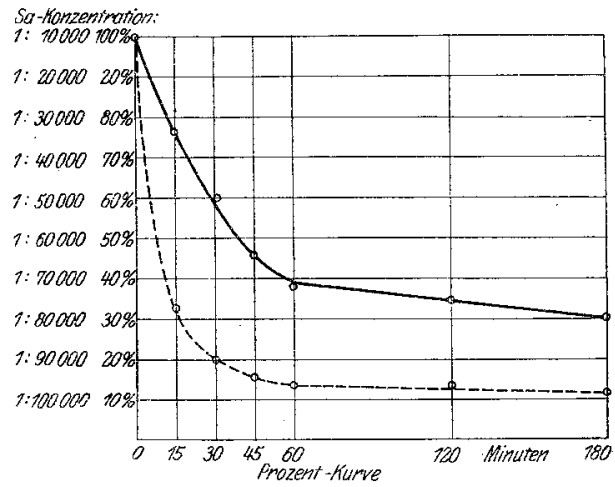

wodurch sich eine mittlere Kurve ergibt, die in nebenstehender Figur dargestellt ist. Nach dieser Kurve ist eine Prozentkurve eingezeichnet (gestrichelt). Diese wurde so gewonnen, daß die Konzentration $x$ : Io00o gleich I00\% Salvarsanmenge gesetzt wurde. Warum gerade dies erlaubt ist, läßt sich ohne weiteres aus den natürlichen Verhältnissen folgern. Nimmt man nämlich die gesamte Blutmenge eines Erwachsenen zu 4,5-5 Litern (und mehr) an, so muß man zu dieser Blutmenge $0,45-0,5$ (und mehr) Salvarsan geben, um eine Konzentration des Salvarsans im Blutserum I : o ooo zu erhalten. Die gefundenen Verdünnungen lassen also sofort den noch im gesamten Blut befindlichen Salvarsangehalt errechnen. Aus der gestrichelten Prozentkurve ist $z u$ entnehmen, daß nach den ersten 15 Minuten post injectionem nur noch etwa 30\% der eingespritzten Salvarsanmenge sich im Blute selbst befinden, also fast $70 \%$ an die übrigen Körperflüssigkeiten und Gewebe abgegeben, zum Teil auch wohl schon ausgeschieden worden sind (Niere, Darm). Der Gesamtgehalt des Blutes an Salvarsan beträgt also nach I5 Minuten nur noch ca. $\mathrm{o}, \mathrm{I}_{5} \mathrm{~g}$. Von diesen sind nach weiteren $\mathrm{I}_{5}$ Minuten kaum noch o, I g (20\% der Gesamtmenge) nachweisbar. Der Konzentrationsabfall setzt sich also weiter fort, allerdings bedeutend langsamer, indem im gleichen Zeitraum nicht wiederum $70 \%$ der noch vorhandenen Menge, sondern nur 30\% davon aus dem Blute verschwinden. Im weiteren Verlauf geht der Konzentrationsabfall mit immer stärkerer Verzögerung vor sich; der Verlauf der Kurve wird dadurch wagerechter. Schließlich verläuft sie lange Zeit der Nullinie fast parallel, bis die Konzentration des Salvarsans im Blutserum nach etwa 3 Stunden so gering geworden ist, daß es sich dem Nachweis mit der Abelinschen Probe entzieht. Wann das Salvarsan endgültig aus dem Blut verschwindet, läßt sich daraus natürlich noch nicht erschließen. Da es im Urin 24 Stunden und länger nachweisbar bleibt, ist anzunehmen, daß auch im Blute so lange 
geringe Mengen Salvarsan kreisen. Das endgültige Verschwinden tritt wohl erst dann ein, wenn das Salvarsanmolekül im Körper völlig abgebaut ist. Was dann noch übrigbleibt - wohl hauptsächlich an celluläre Elemente gebundenes Arsen - kann noch kürzere oder längere Zeit, je nach Empfindlichkeit der Prüfungsmethode, als Arsen nachgewiesen werden.

Zum Schluß möchten wir auf einige praktische Fragen hinweisen, auf die man durch obige Resultate hingelenkt wird. Wohin begibt sich zunächst das Salvarsan so schnell nach der Injektion? Man könnte an eine allgemeine, gleichmäßige Durchtränkung der gesamten Körperzellen und Säfte denken, wenn nicht die Ergebnisse anderer Autoren [ULLMANN ${ }^{15}$ ), STÜHMER ${ }^{16}$ ), dort noch weitere Angaben] mehr für eine Absorption in einzelnen Organen sprächen, während andere (Gehirn) gänzlich frei davon gefunden werden. Geschieht die Absorption in jenen Organen aber so schnell, so ist es ohne weiteres verständlich, daß äie Wirkung in schlechter absorbierenden Körperteilen schwächer ist oder nicht genügend sein kann. Damit wäre eine Erklärung für den Fehlschlag der Therapie sterilisans magna im allgemeinen und für das Zustandekommen beispielsweise von Neurorezidiven gegeben. Da sich aber auf der anderen Seite Neurorezidive durch genügend intensive (besonders kombinierte!) Kurven fast immer vermeiden lassen [ERICH HoFFMANN $\left.\left.{ }^{17}\right)\right]$ - wie weit bei kombinierter Behandlung dabei aber das Quecksilber in Rechnung zu stellen ist, sei dahingestellt - bleibt aufzuklären, eine wie hohe Salvarsankonzentration im Blute notwendig ist, eine ausreichende Wirkung zu verbürgen.

Wir möchten dann noch kurz die Frage streifen, ob eine Maßnahme zur Elimination des einmal eingespritzten Salvarsans, die Brucksche Blutwaschung, ein geeignetes Mittel ist, größere Mengen Salvarsan schnell aus dem Körper wieder zu entfernen. Diese wird ja gewöhnlich so vorgenommen, daß nach einem Aderlaß von roo-20o ccm und mehr Blut $25^{\circ}$ bis $300 \mathrm{ccm}$ und mehr physiologische Kochsalz- oder. Ringersche Lösung intravenös zugeführt werden. Wird der Aderla $\beta$ nicht gleich nach der Injektion gemacht, was wohl in den seltensten Fällen geschieht, so wird dadurch unmittelbar sicher keine nennenswerte Menge Sarlvarsan aus dem Körper herausbefördert. $\mathrm{Ob}$ aber die nachfolgende Infusion von Kochsalzlösung ihrerseits zur Ausscheidung größerer Salvarsanmengen führt, ist uns nicht bekannt. Wir hatten auch bis jetzt keine Gelegenheit, in dieser Richtung Untersuchungen anzustellen. Tatsächlich sind die Erfolge bei akuter Salvarsanvergiftung (Encephalitis haemorthagica) ja recht zweifelhaft. Bei Salvarsandermatitis dagegen, bei der oft eine Besserung zu verzeichnen ist, braucht die Wirkung der Blutwaschung durchaus nicht auf Elimination des Salvarsans als vielmehr auf allgemein entgiftender Wirkung wie bei anderen Dermatosen zu beruhen.

Literatur: 1) F. B́lumenthal, Biochem. Zeitschr. I2, zit. nach ABELIN, Münch. med. Wochenschr. 1912; Nr. 2. - ${ }^{2}$ ) IGERSheimer u. RothmanN, H. S., Zeitschr. f. physiol. Chem. 59, H. 3-4, zit. nach Agelis, Münch. med. Wochenschr. Igr2, Nr. 2. 3) Fischer u. Hoppe, Münch. med. Wochenschr. I9IO, Nr. 29; EHRLICH, Abhandlungen über Salvarsan I, I9II. - 4) BoRnsteIN, Dtsch. med. Wochenschr. IgI I, Nr. $3 .-{ }^{5}$ ) StüMrpke u. SiEGFrIEd, Dtsch. med. Wochenschr. I9I 1, Nr. 39. - ${ }^{6}$ ) LöHLEIN, Münch. med. Wochenschr. I9I I, Nr. I6. - 7 ) HEUSER, Med. Klinik I9II, Nr. I 5. -8) FIScher u. ZeRNIK, Berl. klin. Wochenschr. I9I I, Nr. 34. 9) Burnaschoff, Russki Wratsch IgI2, Nr. I3. - 10) Abeinn, Münch. med.Wochenschr. I9I 2, Nr. 2. - ${ }^{11}$ )RIEBES, Arch. f.Dermatol. u. Syphilis II8, I9I3. - 12) EHRLICH u. BerTuEm, Berichte der dermat. Gesellschaft 45, H. I5, S. 756. I9I2, zit. nach ULIMANN, Wien. klin. Wochenschr. I9I 3, Nr. 23. - 13) AbELIN, Münch. med. Wochenschr. IgI I u. IgI2, Nr. 2. - ${ }^{14}$ ) B. BARKer BEeson u. P. G. AlBRECHT, Arch. of dermatol. a. syphil, Jan. I922. - ${ }^{15}$ ) UlLManN, Arch. f. Dermatol. u. Syphilis I I4. Igr 3. - 16) StüHMER, Arch. f. Dermatol. u. Syphilis i20. 19I4. - ${ }^{17}$ ) ERICH HoffmanN, Dtsch. med. Wochenschr. 19I2, Nr. 7.
„ZUR PHARMAKOLOGIE DES BLUTSERUMS“. Bemerkungen zu dem Referat von H. Handovsky in No. 35 (S. I752) dieser Wochenschrift. Von HERMANN FREUNd (Heidelberg).

Es ist sehr zu begrüßen, daß]HAndovsky die aktuelle Frage nach dem Ineinanderspielen physikalischer und chemischer Bedingungen für den speziellen Fall der pharmakologischen Wirkungen des defibrinierten Blutes zur Diskussion stellt. Die beiden Versuchsreihen, von denen die eine das Wirksamwerden des Serums als rein physikalisches Problem behandelt, während die andere es mit der Bildung wirksamer chemischer Abbauprodukte erklären will, stehen sich wohl nicht so schroff gegenüber wie es aus seinen Ausführungen hervorzugehen scheint; beide Arten von Veränderungen könnten sehr wohl miteinander verknüpft sein. Zwei Möglichkeiten sind denkbar: I. Es könnte - und das gibt HaNDovsky zu - zunächst eine chemische Veränderung eintreten, die Entstehung von Produkten des Eiweißabbaus oder besser des Zellzerfalls; eine solche Milieuänderung wird voraussichtlich den $\mathrm{Zu}$ stand der Serumkolloide verändern, aber auch den der Zellkolloide: Diese letztere Veränder ung tritt als Funktionsänderung, als ,,pharmakologische Wirkung", in Erscheinung, die erstere ist unmittelbar im Serum der Untersuchung zugänglich. Die physikalischen Veränderungen des Serums sind also den Zellwirkungen nebengeordnet und können gleichsam als Modell für das Geschehen an der Zelle dienen. 2. Auch ein anderer Zusammenhang ist, wenn auch ebensowenig bewiesen, denkbar: wie Handovsky anzunehmen scheint, könnte sich primär der physikalische Zustand der Kolloide ändern; veränderte Fällbarkeitsverhältnisse - ,erhöhte Labilität" nach SACHS - würde dann zu dem führen, was wir Vermehrung der Globulinfraktion nennen, damit ist aber höchstwahrscheinlich eine leichtere Spaltbarkeit gegeben, die dann sekundär die Entstehung wirksamer Abbauprodukte begünstigen müßte.

Der Endeffekt wäre in beiden Fällen der gleiche; im Serum müßte nebeneinander eine Vermehrung der Abbauprodukte und eine Veränderung des physikalischen Zustands nachweisbar sein. Die bisher gefundenen Tatsachen schließen sich also nicht aus. Wie steht es nun aber mit der Beweisführung für die primäre Bedeutung des einen oder anderen Vorganges?

Es ist HAnDovsky und $\operatorname{PICK}^{1}$ ) gelungen, durch rein physikalische Zustandsänderungen die gefäßverengernde Serumwirkung zu verstärken. Soweit sie dabei mit Adsorbentien (Kaolin, Fibrin) gearbeitet haben, ist zunächst ein Einwand möglich; mit der in unserem Institut benützten Tierkohle (Mrrck) behandeltes Serum. ist, wie $\mathrm{KIRSTE}^{2}$ ) untersucht hat, praktisch calciumfrei. Dieser wichtige Punkt, der für die Reaktion der Froschgefäße sehr große Bedeutung hat, müßte zunächst geklärt werden.

Des weiteren stützt sich HANDovsKy auf die Dialyseversuche, nach deren Ausfall die vasoconstrictorische Wirksamkeit undialysabel sei. Das steht nun in direktem Widerspruch zu den Befunden DITTLERS ${ }^{3}$ ), der gezeigt hat, daB die darmerregende Wirkung voll in das Dialysat übergeht. Wir haben bisher keinen Anla $B$, für die Darm- und die Gefäßwirkung des Serums verschiedene Ursachen anzunehmen. Auch dieser Widerspruch bedarf noch der Klärung (Adsorption?, andere Dialysemethode?). Handovsky und PICK schließen ferner aus ihren Versuchen, daß die gefäßverengernde Wirkung des Serums an die Albuminfraktion gebunden ist; dem steht aber entgegen, daß beim Kaninchenserum die volle physiologische Wirksamkeit - und zwar an allen untersuchten Objekten - bei der Alkoholfällung in Lösung bleibt, also von den gefällten Eiweißkörpern trennbar ist.

Fügen wir noch hinzu, daß die Wirkungen des Serums große Ähnlichkeit mit dem Wirkungstypus biogener Amine haben man hat sie ja als ,adrenalinähnlich" oder als "histaminlike" bezeichnet -, so erscheint zur Zeit die Annahme besser gestützt, daß doch letzten Endes ,,wirksame Substanzen" die Ursache der Serumwirksamkeit sind, auch wenn ibre chemische Definierung noch aussteht. Aber die noch ungeklärte Frage nach den Beziehungen zwischen chemischen (etwa fermentativen) Abbauvorgängen und physikalischen Zustandsänderungen, wie sie beispielsweise heute in der Blutgerinnungsfrage lebhaft erörtert wird (vgl. Herzfeld und Klinger, Nolf, Stephan), scheint mir besonders wichtig zu sein; nur wenn sie von beiden Seiten her in Angriff genommen wird, dürfen wir hoffen, zum Ziele zu kommen.

Literatur: 1) HANDOVSKY und PICK, Arch. f. exp. Pathol. u. Pharmakol. 71 , I913. - 2) KIRSTE, ebenda, 89, rog. I92x. - 3) DITTLER, Pflitgers Arch. 157, 453. I914. Zeitschr. f. Biol. 89, Iog. rg2x. 